University of Wollongong

Research Online

Faculty of Engineering and Information

Faculty of Engineering and Information

Sciences - Papers: Part A

Sciences

$1-1-2014$

\title{
System implementation of self-mixing interferometry technique-based measurement on Young's modulus
}

Ke Lin

University of Wollongong, k1740@uowmail.edu.au

Yanguang Yu

University of Wollongong, yanguang@uow.edu.au

Zhenghao Liu

University of Wollongong, z|460@uowmail.edu.au

Jiangtao Xi

University of Wollongong, jiangtao@uow.edu.au

Huijun Li

University of Wollongong, huijun@uow.edu.au

See next page for additional authors

Follow this and additional works at: https://ro.uow.edu.au/eispapers

Part of the Engineering Commons, and the Science and Technology Studies Commons

Research Online is the open access institutional repository for the University of Wollongong. For further information contact the UOW Library: research-pubs@uow.edu.au 


\title{
System implementation of self-mixing interferometry technique-based measurement on Young's modulus
}

\begin{abstract}
Young's modulus (denoted by $\mathrm{E}$ ) is an intrinsic and valuable quantity for appropriate estimation of material performance. $\mathrm{E}$ can be obtained from the resonant frequency from the vibration of the tested material sample excited by the external forces. In this paper, we present a system design for determining the parameter by using an optical feedback self-mixing interferometry (OFSMI) system. An OFSMI system consists of a laser diode (LD), a micro-lens and an external target. The material sample to be tested is used as the external target. The vibration of the sample causes the variation of the length of the external cavity, and then causes a modulated laser power of the LD. The modulation contains the vibration information of the tested samples. The OFSMI system can achieve high measurement accuracy with an extremely simple and inexpensive set-up, thus can be thought as a good candidate for the evaluation of material properties. The system design in this work includes the mechanical part for holding and exciting the tested samples and the optical part for picking up the vibration information from the samples. In order to accurately determinate $E$, the exclusive supporting system is used for holding the material sample of specific dimension. The complex waveform of the LD output power is studied and simulated by using our proposed system model. The proposed method is verified by simulation and experiments, and satisfied accuracy of the experiments is achieved.
\end{abstract}

\section{Keywords}

interferometry, mixing, self, technique, implementation, young, system, measurement, modulus

\section{Disciplines}

Engineering | Science and Technology Studies

\section{Publication Details}

Lin, K., Yu, Y., Liu, Z., Xi, J., Li, H. \& Liu, C. (2014). System implementation of self-mixing interferometry technique-based measurement on Young's modulus. Proceedings of SPIE: Optical Metrology and Inspection for Industrial Applications III (pp. 92760K-1-92760K-8). United States: SPIE- International Society for Optical Engineering.

\section{Authors}

Ke Lin, Yanguang Yu, Zhenghao Liu, Jiangtao Xi, Huijun Li, and Cuiling Liu 


\title{
System Implementation of Self-Mixing Interferometry Technique- Based Measurement on Young's Modulus
}

\author{
Ke Lin ${ }^{1}$, Yanguang $\mathrm{Yu}^{* 1}$, Zhenghao Liu ${ }^{1}$, Jiangtao $\mathrm{Xi}^{1}{ }^{1}, \mathrm{Huijun} \mathrm{Li}^{2}$ and Cuiling $\mathrm{Liu}^{3}$ \\ ${ }^{1}$ School of Electrical, Computer and Telecommunications Engineering, University of Wollongong, \\ Northfields Avenue, Wollongong, NSW, 2522, Australia \\ ${ }^{2}$ School of Mechanical, Materials and Mechatronic, University of Wollongong, Northfields Avenue, \\ Wollongong, NSW, 2522, Australia \\ ${ }^{3}$ School of Computer and Information Engineering, Beijing Technology and Business University, No. \\ 33, Fucheng Road, Haidian District, Beijing, 100048, China
}

\begin{abstract}
Young's modulus (denoted by E) is an intrinsic and valuable quantity for appropriate estimation of material performance. E can be obtained from the resonant frequency from the vibration of the tested material sample excited by the external forces. In this paper, we present a system design for determining the parameter by using an optical feedback self-mixing interferometry (OFSMI) system. An OFSMI system consists of a laser diode (LD), a micro-lens and an external target. The material sample to be tested is used as the external target. The vibration of the sample causes the variation of the length of the external cavity, and then causes a modulated laser power of the LD. The modulation contains the vibration information of the tested samples. The OFSMI system can achieve high measurement accuracy with an extremely simple and inexpensive set-up, thus can be thought as a good candidate for the evaluation of material properties. The system design in this work includes the mechanical part for holding and exciting the tested samples and the optical part for picking up the vibration information from the samples. In order to accurately determinate E, the exclusive supporting system is used for holding the material sample of specific dimension. The complex waveform of the LD output power is studied and simulated by using our proposed system model. The proposed method is verified by simulation and experiments, and satisfied accuracy of the experiments is achieved.
\end{abstract}

Keywords: Young's modulus, optical feedback, self-mixing interference

\section{INTRODUCTION}

Material properties, which play a major role in the mechanical behaviour of structures and the properties, have been standardized with traditional static methods (tensile, compressive, flexural, tensional, etc.). But they usually require a separate and dedicated test set-up using high quality averaging strain measurement focusing only on the early part of the stress-strain curve. Additionally, the modulus of some fragile materials is difficult to continuously measure. For example, to reach a measurable deformation, a relatively high stress must be applied, which can influence the structure of the tested materials such as ceramics ${ }^{1}$. Therefore, to realise the potential of certain materials for higher strength and stiffness compared with conventional unreinforced alloys in some areas, it is unsurprisingly that more accurate dynamic methods for measuring the properties are required.

*yanguang@uow.edu.au; phone:+61242218187

Optical Metrology and Inspection for Industrial Applications III, edited by Sen Han, Toru Yoshizawa, Song Zhang, Proc. of SPIE Vol. 9276, 92760K · @ 2014 SPIE · CCC code: 0277-786X/14/\$18 · doi: 10.1117/12.2071360 
Dynamic techniques provide an advantage over static techniques because of ease of specimen preparation, wide variety of specimen shapes and sizes, great precision, and measurement over a wide temperature range. One of the most soughtafter dynamic methods is the resonance methods which are based on the longitudinal or flexural vibration of the sample with simple geometry (a cylinder or a prism with uniform rectangular cross-section, a circular plate). The sample can be excited to vibrate at the resonant frequency either by a driver that continuously varies the frequency of the output signal or by a mechanical impact. Because of the characteristics of instantaneity, easiness, repeatability and non-destructiveness, resonant methods have become one of the mainstream techniques for modulus measurement ${ }^{2-8}$. Recently, optical techniques based resonant methods have been developed for measuring mechanical properties. Comella and Scanlon determined the stiffness and elastic modulus of an array of aluminium cantilever beams that were deflected by atomic force microscopy. Tsai and Fang ${ }^{9}$, and Kang et al. ${ }^{10}$ used the optical methods to measure the resonance frequency of cantilever beam to determine its elastic modulus. Later in 2006, Kang et al. ${ }^{11}$ proposed an elastic modulus evaluation technique of a cantilever beam by vibration analysis based on time-average electronic speckle pattern interferometry.

This paper proposes an intact system design for Young's modulus measurement based on the optical feedback selfmixing interferometry (OFSMI) technique. An OFSMI system consists of a laser diode (LD), a micro-lens and an external target. The material sample ready for experiments is used as the external target. After being excited by an elastic strike with a steel ball, the sample is set into damping vibration, which causes the variation of the length of the external cavity, and then causes a modulated laser power of the LD. The modulation contains the vibration information of the tested samples ${ }^{12}$. As is known that elastic material properties critically affect the vibration behaviour of structures, through applying the reverse of the idea, the vibration behaviour of a particular material can give the material's elastic properties after adequate analysis and investigation on modulation signal are performed.

\section{PRINCIPLE OF MEASUREMENT}

Measurements based on optical techniques have been a powerful means in measuring mode shape of vibration and surface displacement. Nonetheless, the analysis on optical signal processing is very complicated. Self-mixing interferometry (SMI) technique is one of the optical methods developed following this approach ${ }^{13}$. The SMI signal contains large amount of information of the vibration period and displacement of the external target, even some important useful material parameters. An intact OFSMI system is shown in Figure 1. After the laser emitted by the LD is reflected or backscattered from the external target (e.g. a loudspeaker driven with sinusoidal signal) and re-enter into the laser cavity again, it will mix and interfere with the original laser, thus generating a modulated signal, whose frequency and amplitude will change and become a new saw tooth-like complicated waveform, which we call SMI signal ${ }^{14}$. Thus, based on the SMI signal to be processed, the system can be used to retrieve the useful information from the external target, such as Young's modulus.

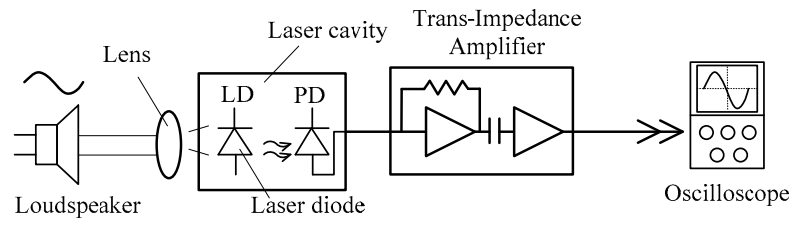

Figure 1. An intact OFSMI system

The SMI has been extensively studied by various scholars and a widely accepted mathematical model based on the Lang Kobayashi equation has been studied. To investigate the analytical relationship between Young's modulus and the modulated laser power, it is rewritten below ${ }^{14-19}$ :

$$
P(t)=P_{0}[1+m G(t)]
$$




$$
\begin{gathered}
G(t)=\cos \left(\phi_{F}(t)\right) \\
\phi_{F}(t)=\phi_{0}(t)-C \sin \left[\phi_{F}(t)+\arctan (\alpha)\right] \\
\phi_{0}(\mathrm{t})=4 \pi y(t) / \lambda_{0}
\end{gathered}
$$

\begin{tabular}{|c|c|}
\hline$t$ & Time index. \\
\hline$P(t)$ & $\begin{array}{l}\text { Power emitted by LD with feedback from } \\
\text { external cavity. }\end{array}$ \\
\hline$P_{0}$ & Laser intensity emitted by the free running LD. \\
\hline$m$ & $\begin{array}{l}\text { Modulation index for the laser intensity } \\
\text { (typically } m \approx 0.001 \text { ). }\end{array}$ \\
\hline$G(t)$ & $\begin{array}{l}\text { SMI function which indicates the influence of } \\
\text { the self-mixing effect on the emitted intensity }\end{array}$ \\
\hline$\phi_{F}(t)$ & $\begin{array}{l}\text { Laser phase with feedback while external target } \\
\text { exists. }\end{array}$ \\
\hline$\phi_{0}(t)$ & $\begin{array}{l}\text { Laser phase without feedback under free } \\
\text { running conditions. }\end{array}$ \\
\hline$C$ & Feedback level factor \\
\hline$\alpha$ & Line-width enhancement factor \\
\hline$y(t)$ & $\begin{array}{l}\text { Vibration displacement function of external } \\
\text { target }\end{array}$ \\
\hline$\lambda_{0}$ & Emitted laser wavelength without feedback \\
\hline
\end{tabular}

where the meaning of parameters in Eq. (1)-(4) are described detailedly as following in Table. 1.

Table 1. Meaning of parameters in Eq. (1)-(4)

In this paper, we will let the external target vibrate in a damping mode with a singular strike hitting on the centre. According to the mathematical model we expounded previously ${ }^{20}$, the motion equation of the point of the target where the laser incident can be written as following:

$$
y(t)=A e^{-a t} \cos (\omega t+\varphi)
$$

where $A, a, \omega, \varphi$ are respectively the amplitude, the attenuating coefficient, the angular frequency and the phase of damping vibration. Hypothetically, if we assume the displacement track $y(t)$ respectively equal to

$$
y_{1}(t)=1.75 \cdot 785 \times 10^{-9} \cdot \sin (2 \pi \cdot 195 t)
$$

and

$$
y_{2}(t)=1.75 \cdot 785 \times 10^{-9} \cdot e^{-60 t} \sin (2 \pi \cdot 195 t) \text {, }
$$


where assign the laser wavelength $\lambda_{0}=785 \times 10^{-9} \mathrm{~m}$. The waveform of $\phi_{0}(t)$ and $G(t)$ can be obtained according to Eq. (1)-(4). As indicated in the Figure 2, the left is shown the corresponding $\phi_{0}(t)$ of oscillating function $y_{1}(t)$ and its SMI signal and the right the corresponding $\phi_{0}(t)$ of damping oscillating function $y_{2}(t)$ and its SMI signal. It is obvious that the fringes represent the amplitude of the vibration, and as the attenuation of the amplitude, the number of fringes gradually reduces. At the same time, the period of simple harmonic vibration in Figure 2 left is practically the same as the period of SMI signal, and so is the damping mode of vibration. Therefore, capitalizing on the characteristics of the SMI signal, the fundamental resonant frequency of the original damping oscillating function can be retrieved if fast Fourier transformation (FFT) is conditionally applied.

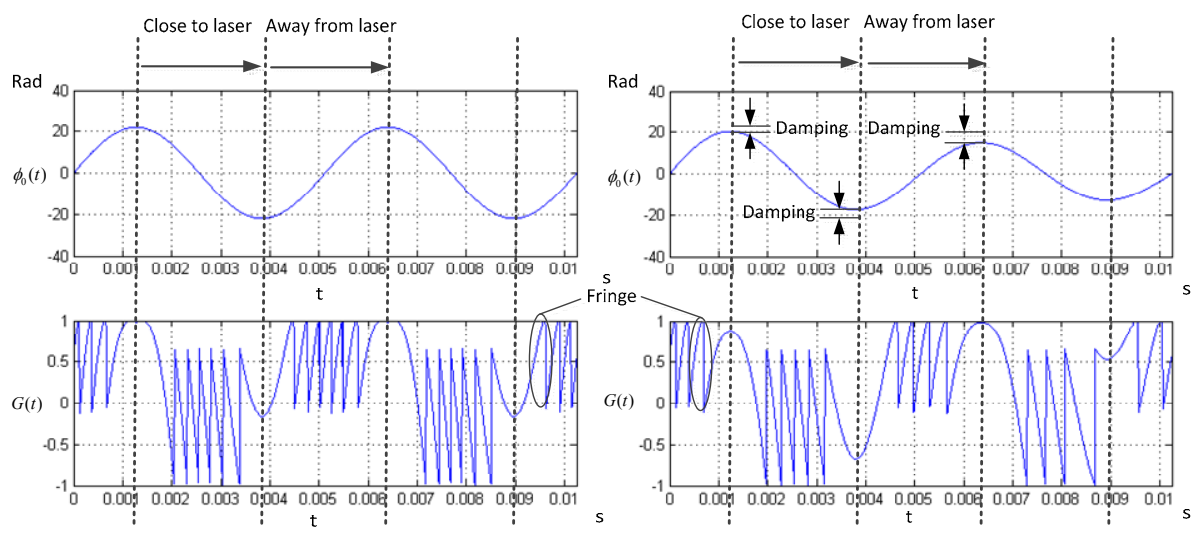

Figure 2 Illustration of SMI signal

\section{DETERMINATION OF YOUNG'S MODULUS}

According to the standard released by ASTM E1876 $6^{21}$, the analytical equation described as follows can represent the mathematical relationship between the fundamental resonant frequency and the Young's modulus. Consequently, the Young's modulus can be readily computed based on the equation shown below.

$$
\begin{gathered}
E=0.9456 \cdot \frac{m f^{2} l^{3}}{b h^{3}} \cdot T_{1}=0.9465 \cdot \frac{\rho f^{2} l^{4}}{h^{2}} \cdot T_{1} \\
T_{1}=1+6.585\left(1+0.752 \mu+0.8109^{2} \mu^{2}\right)(h / L)^{2}-0.868(h / L)^{4} \\
-\left[\frac{8.340\left(1+0.2023 \mu+2.173 \mu^{2}\right)(h / L)^{4}}{1.000+6.338\left(1+0.1408 \mu+1.536 \mu^{2}\right)(h / L)^{2}}\right]
\end{gathered}
$$

where $\mu$ =Poisson's ratio, which can be neglected when certain dimension of the target satisfies and we will talk in the next, other meaning of relevant parameters are described in Table 2. 
Table 2. Meaning of parameters in Eq. (8)

\begin{tabular}{|c|c|}
\hline $\begin{array}{l}E \\
m\end{array}$ & $\begin{array}{l}\text { Young's modulus, Pa. } \\
\text { Mass of the specimen, g. }\end{array}$ \\
\hline$\rho$ & Mass of the specimen, $\mathrm{g} / \mathrm{mm}^{3}$. \\
\hline$f$ & Fundamental resonant frequency in flexure, $\mathrm{Hz}$. \\
\hline$l$ & The length of the specimen, $\mathrm{mm}$. \\
\hline$b$ & The width of the specimen, $\mathrm{mm}$. \\
\hline$h$ & The height of the specimen, $\mathrm{mm}$. \\
\hline$T_{1}$ & $\begin{array}{l}\text { Correction factor for fundamental flexural } \\
\text { mode to account for finite thickness of bar, } \\
\text { Poisson's ratio, etc. }\end{array}$ \\
\hline
\end{tabular}

\section{EXPERIMENTAL WORK}

\subsection{Sample Preparation}

In order to measure the Young's modulus of certain new type isotropic synthetic material or existing material with unknown Young's modulus, necessary verification of current method needs to be made prior to an actual test. Accordingly, in this paper, pure copper is adopted to be the experimental candidate material. Comparison between the actual experimental value and the standard value of Young's modulus of pure copper will be made after tests. Additionally, in according to ASTM E $1876^{21}$, correction factor in the analytical Eq.10 would be different if the comparative result of 20 and the ratio of $h$ and $L$ vary. Since other mechanical properties of the test sample, such as shear modulus and Poison's ratio, are assumed unknown, to preclude any unnecessary iterative process during the computation, the ratio of $h$ and $L$ is manually controlled larger than 20. Thus, the correction factor in Eq.9 can be simplified into the form as following.

$$
T_{1}=1.000+6.585(h / L)^{2}
$$

To assure the reproducibility of the experimental results, two test samples with different thickness $\left(138.35 * 10.12 * 4.35 \mathrm{~mm}^{3}\right.$ and $\left.138.35 * 10.12 * 2.275 \mathrm{~mm}^{3}\right)$ are used.

\subsection{Experimental Setup}

Instead of using Grindosonic MK5 ${ }^{\mathrm{TM}}$ 'Industrial' Instrument proposed in E1876, a system integrated with mechanical and optical parts are introduced. As shown in the Figure 3, the optical system consists of the LD (Thorlabs, HL7851G, $140 \mathrm{~mA}$ ), the temperature and current controller (Thorlabs, ITC102) for accurate control of the working state of the LD, the lens for focusing the laser, the trams-impedance amplifier circuits for initial process of the SMI signal and the oscilloscope (Tektronics, TDS2022B), a readout as well as a sampling media for the wanted processed fringe-numberreducing SMI signal.

The mechanical equipment includes the parts for the support and excitation of the test sample and the recycle procedure for the impacting projectile. Since the vibration modes of the test sample depend on its shape, the place of impact and the type of support, and these modes are well defined for simpler shapes (bars, rods etc.), the supports can be manufactured 
with a sharp knife edge and placed in the nodes of the desired vibration mode 0.224 of the length of the test sample from each end (indicated in Figure 3), thus minimizing the frictional losses at the supports and isolating the test sample from extraneous vibration without restricting the desired mode shape of vibration. In order to keep the reproducibility of the experiments, the impact projectile made of steel is fallen off every time by using a fixed guided tube, whose angle and height can be fine controlled by the fixture as indicated in the picture. Therefore, when each time the projectile freely falls off from the tube, the impact time and force on the test sample which may have influenced the experimental results if varying every time could be negligible during the tests. After excitation, the projectile will be recycled by a magnetic stone ready for next-time use.

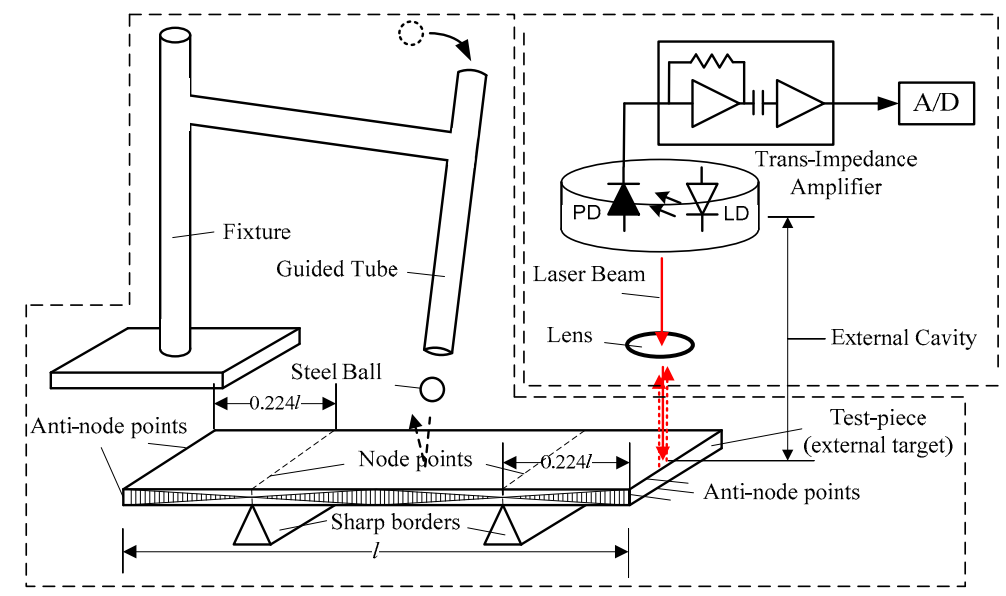

Figure 3 Overall experimental setup diagram

Since optical components are very sensitive to displacement variation of the test sample, to avoid of any undesirable interference from outside, the overall experimental unit is pneumatically set up with an anti-vibration table.

\subsection{Experiment Process}

Each repeatable experiment data sampling was following steps below.

Step 1: Provided the operating current (78mA here) was given bigger enough than the threshold value, in order to capture the maximum displacement of the vibration, the laser incident point was deliberately positioned on the antinode points of the desired vibration mode on one side of the test sample.

Step 2: The distance between the laser cavity and the surface of the sample was adjusted (i.e. the length of the external cavity) to assure the laser incident point was adequately and clearly focused. The oscilloscope was set into a trigger mode waiting for an arising edge of the SMI signal.

Step 3: Then the projectile was leaded into the guided tube, whose angle was adjusted right directed to the centre of the sample, so the ball could freely falls off from the tube and impact the very centre of the sample.

Step 4: The test was repeated until stable data were obtained several times consecutively. The captured picture and coordinate value of the SMI signal were recorded by the oscilloscope.

\section{RESULTS AND DISSCUSION}

In order to ensure the adequateness of sampling points of the signal in a period to be processed by FFT, the sampling rate $250,000 \mathrm{~Hz}$ was set for thinner sample and $500,000 \mathrm{~Hz}$ for the thicker one in the oscilloscope, and signals larger than 
fours periods were obtained. Figure 4 respectively shows the experimental SMI signal and their corresponding FFT results of two samples with different thickness (thinner on the left).

The calculation results are calculated in Table 3 using Eq. 8. The deviation $(\eta)$ is obtained through Eq. 11.

$$
\eta=\frac{\mid \text { calculated modulus value }- \text { standard modulus value } \mid}{\text { standard modulus value }} \times 100 \%
$$

The standard deviation on E measurements for samples with different thickness is respectively about $1.35 \%$ and $1.87 \%$. The calculated E corresponds well with the expected value.
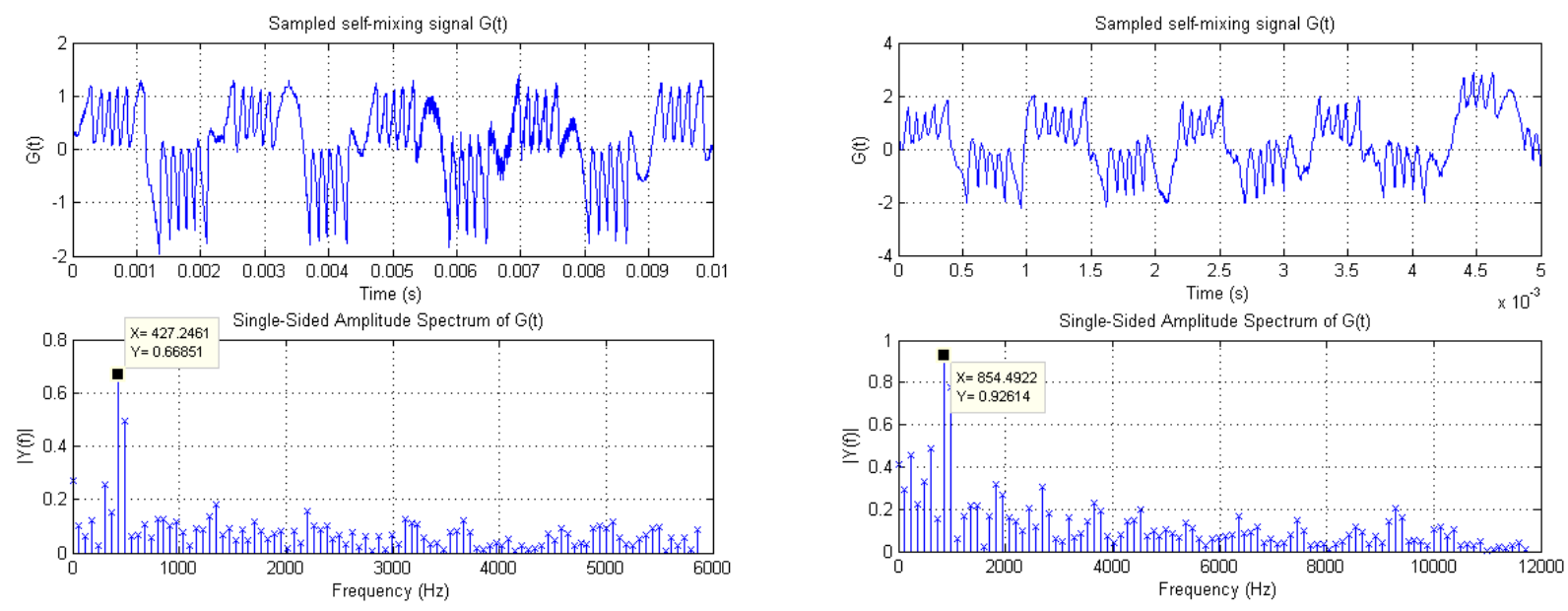

Figure 4. FFT for two sets of experimental signals

Table 3. Young's modulus estimation of steel test sample

\begin{tabular}{cccc}
\hline \hline Thickness (h) & Resonant frequency (f) & Young's modulus (E) & Deviation $(\eta)$ \\
\hline $4.35 \mathrm{~mm}$ & $854.4922 \mathrm{~Hz}$ & $1.06544 \times 10^{11} \mathrm{~Pa}$ & $1.35 \%$ \\
$2.275 \mathrm{~mm}$ & $427.2461 \mathrm{~Hz}$ & $1.05979 \times 10^{11} \mathrm{~Pa}$ & $1.87 \%$ \\
\hline \hline
\end{tabular}

However, it was found that the calculation results are very susceptible to the dimension of the sample. The accuracy could have been more improved by more refined manufacturing process on the test materials. Since the ratio of length and height plays a vital role for calculation the Young's modulus in Eq. (8), it could be seen that from the Figure 4, the thinner the specimen was, the more the dominance of the resonant frequency in FFT was presented.

\section{CONLUSION}

This paper proposes an intact system design for Young's modulus measurement based on the OFSMI technique, which includes the mechanical and optical sections. As the external target vibrates under specific strike and support, the reflected laser signal captured by LD and mixed with the original laser signal contains useful information of vibration. After FFT is applied, the corresponding fundamental resonant frequency can be extracted, which can be used for computing the value of Young's modulus in a high accuracy level. Experiments have proved that OFSMI technique can be well applied in areas of measurement on material properties, especially in a non-destructive and real-time way. 


\section{REFERENCES}

[1] Lord, J. and Morrell, R., "Elastic modulus measurement—obtaining reliable data from the tensile test," Metrologia, 47(2), S41 (2010).

[2] Roebben, G., Basu, B., Vleugels, J., Van Humbeeck, J., and Van der Biest, O., "The innovative impulse excitation technique for high-temperature mechanical spectroscopy," J. Alloy. Compd., 310(1-2), 284-287 (2000).

[3] Kemps, B., De Ketelaere, B., Bamelis, F., Govaerts, T., Mertens, K., Kamers, B., Tona, K., Decuypere, E., and De Baerdemaeker, J., "Development of a Methodology for the Calculation of Young's Modulus of Eggshell using Vibration Measurements," Biosyst. Eng., 89(2), 215-221 (2004).

[4] Suansuwan, N. and Swain, M. V., "Determination of elastic properties of metal alloys and dental porcelains," J. Oral Rehabil., 28(2), 133-139 (2001).

[5] Tognana, S., Salgueiro, W., Somoza, A., and Marzocca, A., "Measurement of the Young's modulus in particulate epoxy composites using the impulse excitation technique," Mat. Sci. Eng. A-Struct., 527(18-19), 4619-4623 (2010).

[6] Stubna, I., Trnik, A., and Vozar, L., "Determination of Young's modulus of ceramics from flexural vibration at elevated temperatures," Acta Acust. United Ac., 97(1), 1-7 (2011).

[7] Atri, R., Ravichandran, K., and Jha, S., "Elastic properties of in-situ processed Ti-TiB composites measured by impulse excitation of vibration," Mat. Sci. Eng. A-Struct., 271(1), 150-159 (1999).

[8] Sakata, M., Kimura, K., and Mizunuma, A., "Measurement of Elastic Moduli From the Impact Sound of Engineering Ceramics and Composites at Elevated Temperatures," J.Am.Ceram.Soc, 78(11), 3040-3044 (1995).

[9] Tsai, H.-C. and Fang, W., "Determining the Poisson's ratio of thin film materials using resonant method," Sensor. Actuat. A-Phys., 103(3), 377-383 (2003).

[10] Kang, X., Tay, C. J., Quan, C., and He, X., "Evaluation of Young's modulus of a vibrating beam by optical method," Opt. Eng., 42(10), 3053-3058 (2003).

[11] Kang, K., Kim, K., and Lee, H., "Evaluation of elastic modulus of cantilever beam by TA-ESPI," Opt. Laser Technol., 39(3), 449-452 (2007).

[12] Scalise, L., Yu, Y., Giuliani, G., Plantier, G., and Bosch, T., "Self-mixing laser diode velocimetry: application to vibration and velocity measurement," IEEE T. Instrum. Meas., 53(1), 223-232 (2004).

[13] Ye, H., Yu, Y., and Guo, C., "Vibration Menasurement Based on Moderate Optical Feedback Self-Mixing Interference," Acta Opt. Sinica, 27(8), 018 (2007).

[14] Yu, Y., Xi, J., and Chicharo, J. F., "Measuring the feedback parameter of a semiconductor laser with external optical feedback," Opt. Express, 19(10), 9582-9593 (2011).

[15] Yu, Y., Qiang, X., Wei, Z., and Sun, X., "Differential displacement measurement system using laser self-mixing interference effect," Acta Opt. Sinica, 19(9) (1999).

[16] Yu, Y., Cheng, M., and Qiang, X., "Self-mixing interference effects in a laser diode with multiple optical feedback," Acta Opt. Sinica, 21(9), 1093-1098 (2001).

[17] Yu, Y., Xi, J., Chicharo, J. F., and Bosch, T. M., "Optical Feedback Self-Mixing Interferometry With a Large Feedback Factor: Behavior Studies," IEEE J. Quantum Electron., 45(7), 840-848 (2009).

[18] Lang, R. and Kobayashi, K., "External optical feedback effects on semiconductor injection laser properties," IEEE J. Quantum Electron., 16(3), 347-355 (1980).

[19] Xi, J., Yu, Y., Chicharo, J. F., and Bosch, T., "Estimating the parameters of semiconductor lasers based on weak optical feedback self-mixing interferometry," IEEE J. Quantum Electron., 41(8), 1058-1064 (2005).

[20] Lin, K., Yu, Y., Xi, J., Fan, Y., and Li, H., "Measuring Young's modulus using a self-mixing laser diode," in SPIE MOEMS-MEMS, 89750B-89750B-8 (2014).

[21] ASTM Standard E 1876 - 01, "Standard Test Method for Dynamic Young's Modulus, Shear Modulus, and Poisson's Ratio by Impulse Excitation of Vibration," ASTM., West Conshohocken, PA (2005). 